\title{
Biochemistry Teaching Through Food Label Analysis
}

Barbosa, N.L. ${ }^{1}$; Correia, Y.M. ${ }^{1}$; Nascimento, M.M. ${ }^{1}$; Oliveira, H.D².

${ }^{1}$ Faculdade Christus, Curso de Fisioterapia, Ceará, Brazil; '2Departamento de Bioquímica e Biologia Molecular, Universidade Federal do Ceará, Ceará, Brazil.

Traditional classes of Basic Biochemistry often rely on memorization of facts and concepts. For this reason, many educators have evaluated new strategies and tools to enrich learning and motivate undergraduate students. As a result of different methodologies used for this purpose, several commited professors are convinced that students learn more effectively if the knowledge they acquire are inserted and contextualized in relevant real-life. This work aimed to report the use of a new strategy to provide first-semester physiotherapy students with a more practical and interesting approach to Biochemistry. The method consisted in the use of food label analysis as an approach to study the structure and function of biomolecules.The activity was developed at Faculdade Christus (located in Fortaleza) using $15 \mathrm{~h}$ of extra classes. Firstly, the students were requested to collect food labels during a week. Secondly, they were teached to read and interpret a food label according the dietary recommendations of World Health Organization and National Health Surveillance Agency - Brazil, contrasting different types of foods with regard to their calories and nutrient content. In this activity, the analysis of structure and function of carbohydrates, lipids and proteins was made to support the understanding of basic concepts. The last activity aimed to organize a healthy breakfast, considering the concepts learned during the food label analysis. The activities were evaluated by the students through written questionaires and informal conversations, indicating good acceptance and approval of this method and showing that this activity is a valid educational tool.

Word Keys: Biochemistry Teaching, Food Labels, Education.

Supported by: Faculdade Christus, Universidade Federal do Ceará. 
This document was created with Win2PDF available at http://www.win2pdf.com. The unregistered version of Win2PDF is for evaluation or non-commercial use only. This page will not be added after purchasing Win2PDF. 